\title{
Link budget investigations for ingestible antenna in MedRadio band
}

\author{
Dimitrios A. Galanis \\ School of Electrical and Computer \\ Engineering, NTUA \\ Iroon Polytechniou 9, 15780, \\ Athens, Greece \\ dgalanis@biosim.ntua.gr
}

\author{
Konstantinos A. Psathas \\ School of Electrical and Computer \\ Engineering, NTUA \\ Iroon Polytechniou 9, 15780, \\ Athens, Greece \\ kpsathas@biosim.ntua.gr
}

\author{
Konstantina S. Nikita \\ School of Electrical and Computer \\ Engineering, NTUA \\ Iroon Polytechniou 9, 15780, \\ Athens, Greece \\ knikita@ece.ntua.gr
}

\begin{abstract}
Ingestible Medical Devices (IMDs) have given a strong boost to the health sector by creating new horizons in the prevention, monitoring and treatment of diseases in the gastrointestinal (GI) tract. In this study, we numerically assess the telemetry link between an ingestible antenna and an on-body antenna which acts as a repeater. Both antennas can be incorporated in a complete Wireless Capsule Endoscopy (WCE) system. Two different scenarios are examined to assess the performance of the ingestible antenna inside the small intestine of an anatomical human model and the link with the on-body antenna. The ingestible antenna is placed in vertical and horizontal position inside the small intestine. It is found that a reliable communication link can be established, regardless the antenna orientation inside the small intestine, for a net input power of the ingestible antenna significantly lower than the maximum allowable power recommended by the IEEE safety guidelines.
\end{abstract}

\section{Categories and Subject Descriptors}

B.8.2 [performance and reliability]: Performance Analysis and Design Aids.

\section{General Terms \\ Design, Performance.}

\section{Keywords}

Biocompatible ingestible antennas, wireless capsule endoscopy, on-body antennas, medical device radiocommunication (MedRadio) services, biomedical telemetry.

\section{INTRODUCTION}

Nowadays, people suffer from gastrointestinal (GI) diseases due to changes in their diets, environmental pollution, and mental stress. Gastrointestinal complications include intestinal cancer, intestinal tumors, peptic disease, Crohn's disease and inflammatory bowel [1]. Traditional wired gastrointestinal endoscopic systems that are most commonly used can induce discomfort or even pain in the patient and are related with limited test coverage of the GI tract [2]. Moreover, due to the fact that the small intestine is long and twisted, it is almost impossible to access it by means of conventional wired endoscopy which has long considered it as the "black box" of the GI system. In the last few years, Wireless Capsule Endoscope (WCE) systems [3], [4] have been proposed for diagnosis and medical treatment in modern medicine in order to overcome these limitations.

WCE systems include an ingestible capsule, sensors and a data recorder. The capsule, which is swallowed by the patient, passes through the digestive tract collecting images and/or physiological parameters and wirelessly transmits them. The sensors, which are connected with the data recorder, receive transmission data from the capsule and transfer them to the data recorder that is worn by the patient during the study. These sensors are placed on the patient as a sensor belt or a sensor array around the abdominal area. After the study, the data are downloaded onto a computer where are then viewed and interpreted by a specially trained gastroenterologist. Commercial Ingestible Medical Devices (IMDs) commonly use the low-frequency ISM band of $433 \mathrm{MHz}$. Low-frequency IMDs have been shown to be favorable for short range communication due to less absorption by human tissues [5], [6].

One of the most important components of IMDs is the ingestible antenna, used to exchange data with the external device. A major ingestible antenna design challenge is to achieve a wide $10 \mathrm{~dB}$ bandwidth while still maintaining miniature size and adequate radiation performance. Enhanced bandwidth is a crucial aspect given the requirement for high data rates, and the detuning effects which might be caused from the inhomogeneous human environment. Given the miniature dimensions of the capsule and the limited space left after the integration of sensors, cameras, LEDs, electronics and batteries, antenna size becomes a significant concern for the designer. Omni-directivity is also a pre-requisite for ingestible antennas in order to maintain their performance regardless of their position and orientation which are, in principle, unknown.

In this study, we suggest the use of an on-body dual-band antenna which receives the weak signals of the ingestible antenna in the MedRadio band, (Medical Device Radiocommunications Service, 401-406 MHz) and further retransmits them in the ISM band (Industrial, Scientific and Medical, 2400-2480 MHz) to an exterior monitoring device. The link between the ingestible and the on-body antenna in MedRadio band is examined, evaluating two different scenarios considering the polarization of the ingestible antenna inside the small intestine. 


\section{MODELS AND METHODS}

\subsection{Antenna models}

The proposed ingestible antenna features a PIFA design philosophy and was introduced by the authors in [7]. The conformal radiating patch (Figure 1(a)) features seven meanders to increase the effective length of the current flow. The meanders are inserted to assist with antenna miniaturization without significantly decreasing the antenna surface which is crucial for its radiation performance. Moreover, a shorting pin is used for miniaturization purposes.

The geometry of the proposed dual-band on-body antenna (Figure 1(b)) was introduced by the authors in [8]. The on-body antenna exhibits rectangular shape and consists of rectangular ground and triangular patch planes.

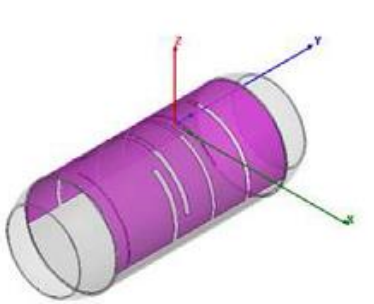

(a)

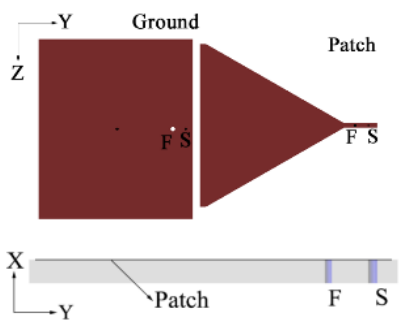

(b)
Figure 1. (a) Geometry of the proposed ingestible antenna. (b) Geometry of the proposed on-body antenna.

\subsection{Tissue models and numerical methods}

Ingestible antenna design and performance investigations are performed by considering the antenna placed in the center of the cubic homogeneous model of Figure 2. The cube is filled with tissue equivalent material for $402 \mathrm{MHz}$ (permittivity, $\varepsilon_{\mathrm{r}}=57.2$, conductivity, $\sigma=0.93 \mathrm{~S} / \mathrm{m}$ [9]), and exhibits dimensions of $100 \mathrm{~mm}$ x $100 \mathrm{~mm}$ x $100 \mathrm{~mm}$. The use of an anatomical human model (part of the Virtual Population by IT'IS [10]) is considered for antenna performance and telemetry link investigations in a more realistic scenario (Figure 3(a)).

Finite Difference Time Domain (FDTD) simulations are carried out using the SEMCAD $X$ software by SPEAG [11]. For electromagnetic simulations, a minimum spatial resolution of 6.55 $\mathrm{x} 12.75 \mathrm{x} 48.65 \mu \mathrm{m}^{3}$ and maximum spatial resolution of $4.09 \mathrm{x}$ $32.13 \times 3.96 \mathrm{~mm}^{3}$ in the $\mathrm{x}, \mathrm{y}$, and $\mathrm{z}$ directions is chosen for MedRadio band.

\section{RESULTS AND DISCUSSION}

\subsection{Design approach}

A cubic model is adopted (Figure 2) for the ingestible antenna optimization phase and the assessment of its resonance performance inside the GI tract. The cubic model exhibits much faster computational time compared to the more complex anatomical model, which is used here only for the radiation and safety performance evaluation and telemetry link assessment. After maintaining the ingestible antenna resonance performance inside the cubic model, the antenna is placed inside the anatomical human model (Figure 3(b)). Simulations are carried out to compare the resonance performance of the ingestible antenna between the aforementioned models. In addition, radiation and safety performance of the ingestible antenna are evaluated. Moreover, the on-body antenna is considered in free space and at a distance of 15 $\mathrm{mm}$ (Figure 3(c)) from the abdomen of the human model of Figure $3(\mathrm{a})$, in order to assess the effect of the human body in the on-body antenna performance. The distance of $15 \mathrm{~mm}$ is chosen to take into consideration the presence of human clothes in a realistic scenario [8].

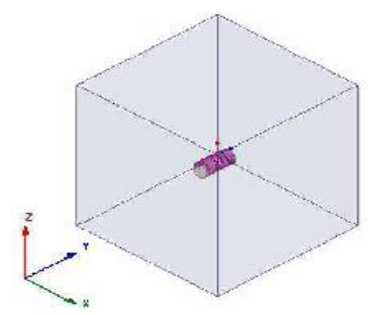

Figure 2. Cubic model used to design the ingestible antenna.

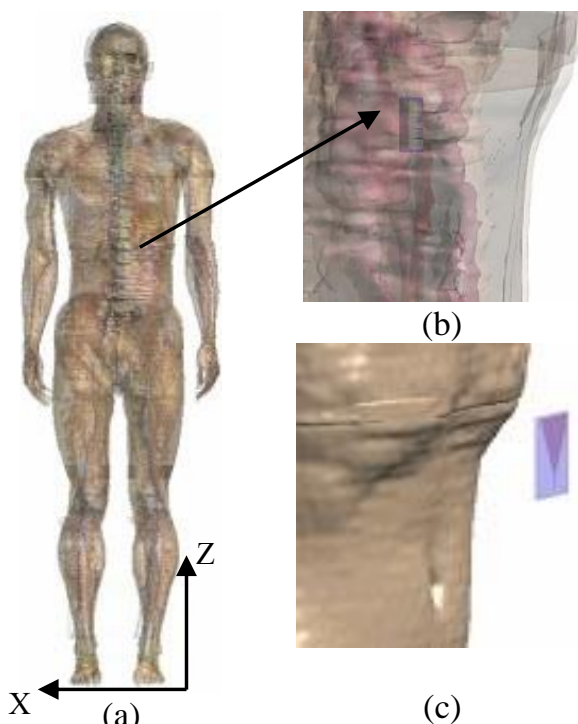

Figure 3. (a) Duke anatomical model. (b) Ingestible antenna inside the small intestine of the anatomical model. (c) On-body antenna placed $15 \mathrm{~mm}$ from the abdomen of the anatomical model.

\subsection{Design methodology validation}

The frequency response of the reflection coefficient $\left(\left|S_{11}\right|\right)$ of the ingestible antenna inside the cubic model and the anatomical model is shown in Figure 4. The ingestible antenna resonates inside the cubic model at $402 \mathrm{MHz}$ with a reflection coefficient magnitude $\left(\left|\mathrm{S}_{11}\right|\right)$ of $-43.62 \mathrm{~dB}$ and a $10 \mathrm{~dB}$-bandwidth of $29.12 \mathrm{MHz}$ (or $7.21 \%$ of MedRadio) and inside the anatomical model at 401.5 $\mathrm{MHz}$ with a reflection coefficient magnitude (|S11|) of $-32.62 \mathrm{~dB}$ and a wide $10 \mathrm{~dB}$-bandwidth of $30.27 \mathrm{MHz}$ (or $7.5 \%$ of MedRadio).

The 3D far-field radiation pattern inside the cubic model (Figure $5(\mathrm{a})$ ) is almost omni-directional, and the maximum gain is found to be equal to $-33.56 \mathrm{~dB}$. The directivity of the antenna can be attributed to the conformity and the asymmetry of the radiating patch. Inside the anatomical model the far-field radiation pattern of the ingestible antenna (Figure 5(b)) is directional and asymmetric due to inhomogeneous structure of the human body. Assuming a net input power of $1 \mathrm{~W}$ incident to the ingestible antenna, the maximum $1 \mathrm{~g}$-averaged $\left(\max . \mathrm{SAR}_{1 \mathrm{~g}}\right.$ ) and $10 \mathrm{~g}$-averaged (max. $\mathrm{SAR}_{10 \mathrm{~g}}$ ) specific absorption rate (SAR) values are summarized in Table 1. Therefore, in order to guarantee conformance with the IEEE C95.1-1999 (1g-avg SAR $\leq 1.6 \mathrm{~W} / \mathrm{kg}$ [12]) and IEEE C95.12005 (10g-avg SAR $\leq 2 \mathrm{~W} / \mathrm{kg}$ [12] safety standards, the maximum allowable net input power has to be limited according to Table 1 . 


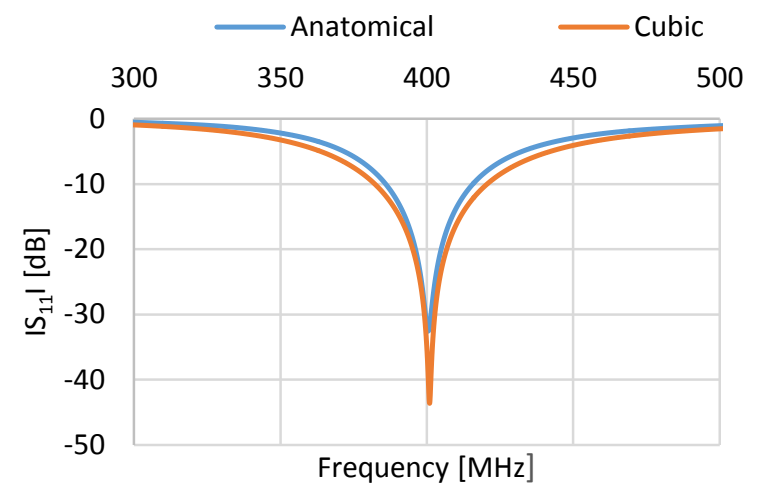

Figure 4. Reflection coefficient frequency response of the ingestible antenna inside the cubic model and the anatomical model.

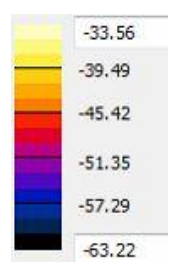

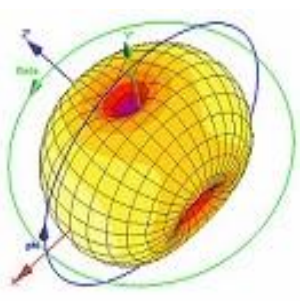

(a)

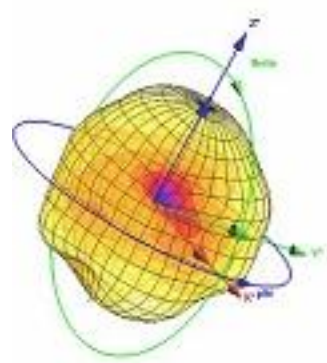

(b)
Figure 5. Radiation (3-D far field radiation pattern) performance $[\mathrm{dBi}]$ of the ingestible antenna (a) in the cubic model, (b) in the anatomical model.

The reflection coefficient $\left(\left|S_{11}\right|\right)$ frequency response of the on-body antenna is shown in Figure 6. The on-body antenna resonates at $403.03 \mathrm{MHz}$ in free space with a reflection coefficient magnitude $\left(\left|\mathrm{S}_{11}\right|\right)$ of $-22.16 \mathrm{~dB}$ and a $10 \mathrm{~dB}$-bandwidth of $6.85 \mathrm{MHz}$.

Table 1. Safety performance of the ingestible antenna operating at $403.5 \mathrm{MHz}$.

\begin{tabular}{|c|c|c|c|c|}
\hline & $\begin{array}{c}\text { max. } \\
\text { SAR }_{1 \mathrm{~g}} \\
{[\mathrm{~mW} / \mathrm{g}]} \\
\mathrm{P}_{\text {in }}=1 \mathrm{~W}\end{array}$ & $\begin{array}{c}\mathbf{P}_{1999} \\
{[\mathrm{dBm}]}\end{array}$ & $\begin{array}{c}\max . \\
\text { SAR }_{10 g} \\
{[\mathrm{~mW} / \mathrm{g}]} \\
\mathbf{P}_{\text {in }}=1 W\end{array}$ & $\begin{array}{c}\mathbf{P}_{2005} \\
{[\mathbf{d B m}]}\end{array}$ \\
\hline $\begin{array}{l}\text { Cubic } \\
\text { Model }\end{array}$ & 314 & 7.06 & 80.5 & 13.95 \\
\hline $\begin{array}{c}\text { Anatomical } \\
\text { Model }\end{array}$ & 282 & 7.53 & 77.2 & 14.13 \\
\hline
\end{tabular}

When placed in the proximity of the human body, the resonance frequency is slightly shifted to $400.21 \mathrm{MHz}$ with a reflection coefficient magnitude (|S11|) of $-23.51 \mathrm{~dB}$ and a $10 \mathrm{~dB}$-bandwidth of $5.72 \mathrm{MHz}$. This is to be expected and is attributed to the lower effective permittivity of the antenna due to the biological tissues.

The radiated far-field gain radiation pattern (Figure 7(b)) when the antenna is placed at a distance of $1.5 \mathrm{~cm}$ from the human body is almost omni-directional in the $\mathrm{XZ}$ plane, and the maximum farfield gain value is found to be equal to $-22.79 \mathrm{~dB}$.

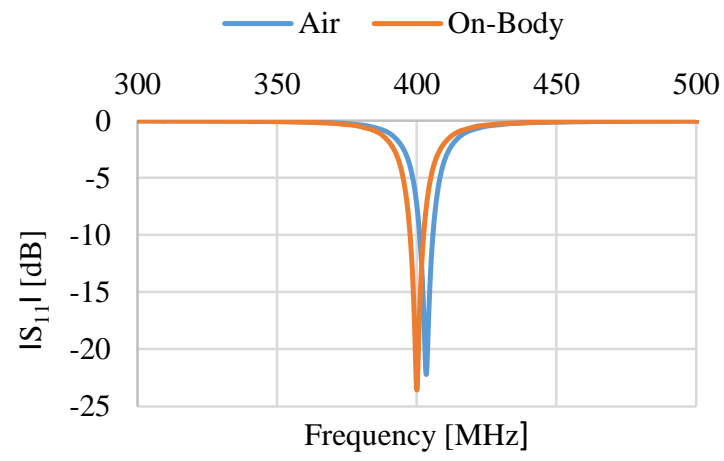

Figure 6. Reflection coefficient frequency response of the onbody antenna in the free space and in the anatomical model in the MedRadio band.

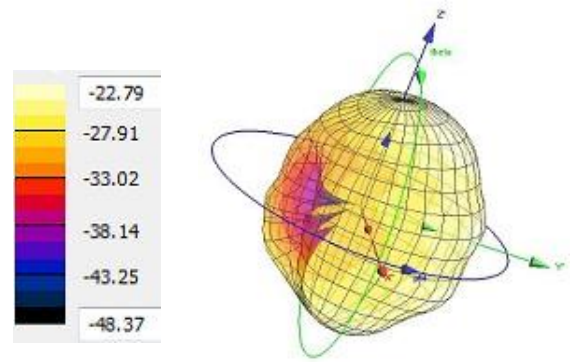

Figure 7. Radiation (3-D far field radiation pattern) performance $[\mathrm{dBi}]$ of the on-body antenna when placed in the proximity of the anatomical human model.

\subsection{Telemetry link investigation}

In order to perform communication link investigations between the two antennas, two scenarios are studied. In both scenarios, the onbody antenna is placed parallel to the abdomen of the human model at a distance of $15 \mathrm{~mm}$ as shown in Figure 8. In the first scenario (Figure 8(a)) the capsule is placed inside the small intestine of the human model parallel to the on-body antenna in such a way that the radiation patch of the ingestible antenna is facing the abdomen of the human model. In the second scenario (Figure 8(b)), the capsule is placed again in the small intestine of the human model with the difference that the capsule is placed vertically to the on-body antenna with the radiating patch of the ingestible antenna facing the upper body of the human model. This placement is chosen in an attempt to investigate the worst communication link scenario.
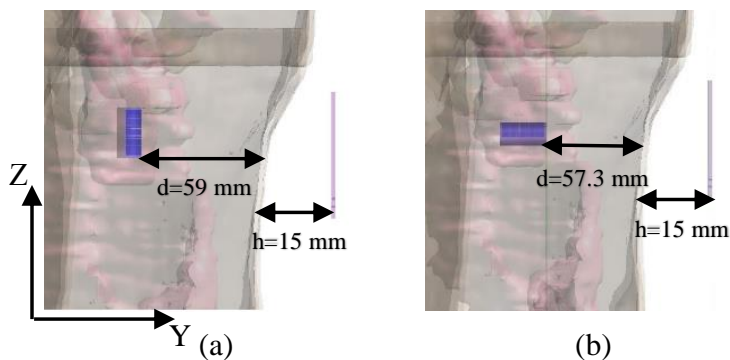

(b)

Figure 8. Link investigation scenarios between the ingestible and the on-body antenna in the anatomical model. (a) Vertical polarization, (b) Horizontal polarization.

For both scenarios the corresponding transmission coefficient is illustrated in Figure 9. In the first scenario, the transmission 
coefficient between the ingestible and the on-body antenna is calculated as $-63.85 \mathrm{~dB}$ at $402.43 \mathrm{MHz}$ while in the second scenario is equal to $-68.44 \mathrm{~dB}$ at $400.92 \mathrm{MHz}$. As expected, the second scenario results in a decrease of $4.59 \mathrm{~dB}$ compared to the first one. The difference in the resonance frequency is attributed to the slightly shifted resonance frequency of the ingestible antenna due to its position. Assuming a transceiver connected to the on-body antenna with a sensitivity of $-96 \mathrm{dBm}$ (Zarlink ZL70102 [13]) a reliable link between the two antennas for both scenarios can be achieved by limiting the ingestible antenna input power to -27.56 $\mathrm{dBm}$ (or $1.75 \mu \mathrm{W}$ ), thus, limiting the EM exposure and preserving valuable battery life for the rest of the components of the ingestible capsule. Considering the maximum allowed power $\mathrm{P}_{1999}=7.53$ $\mathrm{dBm}$ (or $5.67 \mathrm{~mW}$ ), the proposed repeater configuration is found to achieve a reduction in the ingestible capsule power by a factor of 3240 .

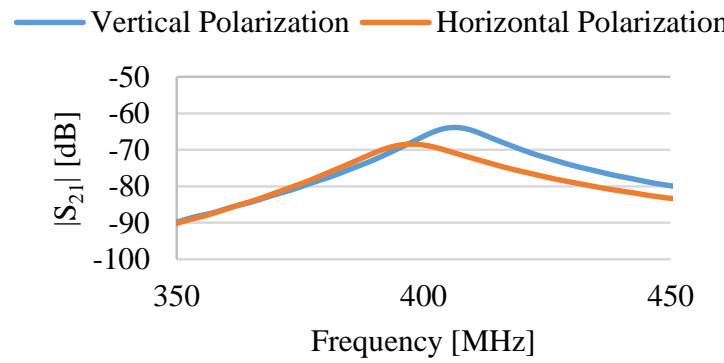

Figure 9. Transmission coefficient $S_{21}$ of ingestible and onbody antenna inside the anatomical model.

\section{CONCLUSION}

In this paper, link investigations were carried out between an ingestible antenna and an on-body repeater antenna. The reflection coefficient frequency response of the ingestible antenna was studied inside a cubic model and an anatomical model in the MedRadio band. Numerical results for both configurations are almost identical with a shift of $0.5 \mathrm{MHz}$ in the resonance frequency and a difference of $1.15 \mathrm{MHz}$ in the $10 \mathrm{~dB}$-bandwidth. For communication link investigations, two different scenarios were studied with the ingestible antenna placed inside the small intestine of the anatomical model with different polarization and the on-body antenna placed at a distance of $15 \mathrm{~mm}$ from the abdomen of the anatomical model. It was found that regardless the position of the ingestible antenna inside the small intestine a robust and reliable link can be established. Assuming the worst case scenario and according to the transmission coefficient the communication link can be reliable for a net input power of $-27.56 \mathrm{dBm}$ of the ingestible antenna, which is significantly lower than the maximum allowable power recommended by the IEEE safety standards.

\section{ACKNOWLEDGMENTS}

This work has been co-financed by the European Union (European Social Fund, ESF) and national funds, under the project ARISTEIA DEM-II- MED ("Implantable and Ingestible Medical Devices (IIMDs): Optimal- Performance-Oriented Design and Evaluation Methodology") and the project THALES ("Multilevel assessment on biological effects of radiofrequency electromagnetic waves (mBioRF)").The authors would like to thank Schmid \& Partner Engineering AG (SPEAG) for providing the license for SEMCAD$\mathrm{X}$ software through SEMCAD X for Science agreement.

\section{REFERENCES}

[1] B. Kim, S. Lee, J. H. Park, and J. O. Park, "Design and Fabrication of a Locomotive Mechanism for Capsule-Type Endoscopes Using Shape Memory Alloys (SMAs)", IEEE/ASME Transactions on Mechatronics, Vol. 10, No. 1, pp. 77-86, 2005.

[2] S. H. Lee, J. Lee, Y. J. Yoon, S. Park, C. Cheon, K. Kim and S. Nam, "A Wideband Spiral Antenna for Ingestible Capsule Endoscope Systems: Experimental Results in a Human Phantom and a Pig", IEEE Transactions on Biomedical Engineering, Vol. 58, No. 6, pp. 1734-1741, 2011.

[3] A. Kiourti, K. A. Psathas and K. S. Nikita, "Implantable and Ingestible Medical Devices with Wireless Telemetry Functionalities: A Review of Current Status and Challenges", Bioelectromagnetics, Vol. 35, No. 1, pp. 1-15, 2014.

[4] K. S. Nikita, N. Bourbakis, and A. Karargyris, "Ingestible Health Care System Paradigm for Wireless Capsule Endoscopy", in Handbook of Biomedical Telemetry, John Wiley \& Sons, Inc., Hoboken, NJ, USA, 2014.

[5] L. Xu, M. Q. H. Meng, H. Ren and Y. Chan, "Radiation Characteristics of Ingestible Wireless Devices in Human Intestine Following Radio Frequency Exposure at 430, 800, 1200 , and $2400 \mathrm{MHz}$ ", IEEE Transactions on Antennas and Propagation, Vol. 57, No. 8, pp. 2418-2428, 2009.

[6] M. R. Basar, F. Malek, K. M. Juni, M. I. M. Saleh, M. S. Idris, L. Mohamed, N. Saudin, N. A. M. Affendi and A. Ali, "The Use of a Human Body Model to Determine the Variation Path Losses in the Body Channel in Wireless Capsule Endoscopy", Progress In Electromagnetics Research, Vol. 133, pp. 495-513, 2013.

[7] K. A. Psathas, A. Kiourti, and K. S. Nikita, "A Novel Conformal Antenna for Ingestible Capsule Endoscopy in the MedRadio Band", In Proceedings of Progress In Electromagnetics Research Symposium, pp. 1899-1902, Sweden, 12-15 August, 2013.

[8] A. Kiourti, J. R. Costa, C. A. Fernandes and K. S. Nikita, "A Broadband Implantable and a Dual Band On-Body Repeater Antenna: Design and Transmission Performance", IEEE Transactions of Antennas and Propagation, Vol. 62, No. 6, pp. 2899-2908, 2014.

[9] FCC. Washington D.C., USA, Federal Communication Commission, "Evaluating Compliance with FCC Guidelines for Human Exposure to Radiofrequency Electromagnetic Fields", 2001 [Online]. Available: http://www.fcc.gov

[10] A. Christ, et al., "The Virtual Family--development of surface-based anatomical models of two adults and two children for dosimetric simulations," Physics in Medicine and Biology, Vol. 55, pp. 23-38, 2010.

[11] SEMCAD X, Schmid \& Partner Engineering AG (SPEAG), Version 14.8 Aletsch. [Online] Available: http://www.speag.com/products/semcad

[12] IEEE Standard for Safety Levels With Respect to Human Exposure to Radiofrequency Electromagnetic Fields, $3 \mathrm{kHz}$ to $300 \mathrm{GHz}$, IEEE standard C95.1, 1999, 2005.

[13] Microsemi, ZL70102 Medical Implantable RF Transceiver MICS RF Telemetry [Online] Available: http://ulp.zarlink.com/zarlink/hs/82_ZL70102.htm 